\title{
My Friend Kolya Delone
}

\author{
D. F. Zaretskii \\ Russian Scientific Center Kurchatov Institute, Moscow, 123182 Russia \\ e-mail:zaretcky@mail.ru \\ Received February 24, 2009
}

PACS numbers: $01.60 .+\mathrm{q}$

DOI: $10.1134 / \mathrm{S} 1054660 \mathrm{X} 09160087$

I met Kolya long time ago. I think I know him, since I remember myself. We were students at the Radio Faculty, Moscow Energy Institute, where N.I. Akhiezer (Academician of the Academy of Sciences of Ukraine) and Prof. B.M. Yavorskii were our lecturers in Mathematics and Physics, respectively. Acad. Akhiezer formulated complicated mathematical problems and the students who were the first to solve them were allowed to skip exams. Kolya was one of the first. We studied at the Radio Faculty for about one and a half years. Then, Kolya entered Moscow Engineering Physics Institute and I became the student of the Physics Faculty, Moscow State University. After the graduation, we lost each other for a long time. Once, I met Kolya at the Bezengi mountaineering camp. He was an experienced mountain climber whereas my friends and I took part in a mountain tour. Each camp has its own traditions: in the evening, people sing sitting around fire, drink tea, and tell stories. I remember the beautiful place with the noise of the river, stars in the sky, and moonlight on the Bezengi wall. In the morning, Kolya went to the mountains and we continued our tour.

The next meeting took place when I intended to quit nuclear physics. My coworker V.P. Krainov made the same decision and I helped him to meet Kolya, who could assist in laser science. Indeed, the discussions helped in formulating the appropriate problem related to the multiphoton processes in the presence of strong laser fields. Krainov was the first to quit the nuclear physics. His long-term cooperation with Kolya has yielded multiple publications and monographs. I am also grateful to Kolya for the changes in my scientific activities.

Next time, we met each other when I acquired substantial knowledge in laser physics. In the 1980s, the Seminars on Laser Physics were organized at the Uzh- gorod Univeristy. The experts in laser physics from Uzhgorod, Kiev, and Voronezh Universities; General Physics Institute; Moscow Engineering Physics Institute; and Moscow State University took part in the Seminar. Kolya was one of the key persons. In the informal atmosphere, we could discuss topical problems during sightseeing. Kolya tried to interpret the results obtained at the Uzhgorod University by Bondar' and Suran who measured the yield ratio of the singly and doubly charged ions for several laser-irradiated atoms. The doubly charged ion yield was significantly higher than the predictions of the perturbation theory. Several concepts were proposed to account for such an anomalous behavior. In spite of hot discussions, we failed to find an interpretation. I am not sure that such an interpretation exists at present.

I attended the Seminar headed by Kolya at the General Physics Institute. I liked the meeting for democratic traditions and a high scientific level. Being the Head of the Seminar, Nikolai Borisovich displayed unique abilities in the interpretation of physical effects. He could clearly formulate the correct conclusions and discuss them with the presenting author. In one of the presentations, I assumed that the absorption of laser energy in a cluster might differ from the absorption in macroscopic plasma due to a significant contribution of the absorption upon the electron collision with the cluster surface. By that time, I could not provide a theoretical analysis and only estimations were available. Several participants had negative comments but Kolya's positive conclusion has stimulated the further study. Thus, I prepared several publications and defended the thesis. Several participants of the Seminar on Multiphoton Processes work at the leading laser laboratories in Europe and USA.

I was fortunate to meet and work with the brilliant physicist Kolya Delone whom I will remember forever. 